\title{
Influence of Nanosecond Pulse Bursts at High Repetition Rates on Ablation Process
}

\author{
John Linden*1,2, Sharona Cohen ${ }^{2}$, Yuval Berg ${ }^{2,3}$, Zvi Kotler $^{2}$, and Zeev Zalevsky ${ }^{1}$ \\ ${ }^{1}$ Department of Electro-Optics, Faculty of Engineering, Bar Ilan University, Israel \\ ${ }^{2}$ Additive Manufacturing Research Group, Orbotech Ltd., Israel \\ ${ }^{3}$ Department of Physical Electronics, Faculty of Engineering, Tel Aviv University, Israel \\ ${ }^{*}$ Corresponding author's e-mail address:john.linden@orbotech.com
}

\begin{abstract}
Nanosecond laser pulsed ablation is a common technique for micromachining of microelectronics. Recent laser technologies use temporal shaping of single pulses to create 'pulse bursts' of several short consecutive pulses at several times the laser repetition rate with a reduced peak power, achieving significantly higher average powers. In this study we tested the effects of pulse bursts on ablation of multilayer PCB. We show that by implementing temporal beam shaping, we were able to increase the throughput by a factor of $40 \%$. To gain a deeper insight of the laser mater interactions of ablation with pulse bursts, we studied the plasma emissions and monitor the process with a time resolution of several nanoseconds. These results demonstrate the importance of temporal pulse shapes for laser micromachining in the microelectronics industry.
\end{abstract}

DOI: $10.2961 /$ jlmn.2021.01.2003

Keywords: nanosecond ablation, plasma dynamics, temporal pulse shaping, microelectronics, pumpprobe microscopy

\section{Introduction}

As modern-day electronics advance to more compact, robust, and flexible devices, the industry is striving to provide solutions for the demands of the next generation. One of the solutions currently adopted is the use of nanosecond UV lasers for via (vertical interconnect access) formation[1-3]. This choice driven by the calibrated absorption in the interlayers of polyamide and prepreg, small heat affected zones (HAZ) and small processing diameters, allow for high accuracy drilling of HDI (High Density Interconnects) in FPCBs (Flexible Populated Circuit Boards). As the defining market advantage in the industry is high throughput[4,5], pulse bursts are being introduced which allow for high average powers to be adopted in MOPA (Master Oscillator Power Amplifier) lasers without damaging optical and mechanical components[6-8].

As MOPA laser systems are being implemented with advanced electronics, the time resolution for controlling the amplification of short-pulsed lasers has increased to the nanosecond level[9,10]. The common use of a MOPA system in a laser is to produce a high-power short laser pulse with great accuracy while allowing the adjustment of pulse width, pulse energy, and pulse repetition rates (PRR) to be individually altered. The introduction of temporal pulse shaping, and pulse bursts is another example permitted by the MOPA structure where the single pulse width may be increased and it's intensity modulated by controlling the temporal gain profile. As the temporal profiles have been shown to affect the results of laser ablation, the pulse-bursts have shown more intriguing results.

In this study we explore the effect of ablation parameters in the drilling of copper layers when using pulse bursts. Ablation parameters in the radiative transfer process is reviewed in references [11,12]. The experimental results show a strong variation of threshold fluence with the laser pulse shape. Realizing these pulse shapes can prove an advantage for dramatically increasing process throughput.

To explore the interactions between laser pulses when using laser pulse bursts with high repetition rates similar to [13-16], a fast-imaging system was constructed to monitor the ablation process. The study focus is on the ablation effects of beam shaping at PRRs on microelectronic substrates with nanosecond UV pulses. The insights we provide will affect the adoption and optimization of temporal pulse shaping for laser micromachining in the microelectronics industry.

\section{Materials and Methods}

The experimental setup used to develop the results presented in this study include several separate apparatuses. All originate with a Boreas Eolite, high-power nanosecond fiber $343 \mathrm{~nm}$ (UV) laser capable of adjusting its single pulse width continuously from 1-30ns. The laser source employs a fiber MOPA allowing for temporal pulse shaping with a programmable resolution of $1 \mathrm{~ns}$. In this study several different temporal pulse profiles were tested with pulsewidths ranging from $5 \mathrm{~ns}$ to $20 \mathrm{~ns}$ with repetition rate of $50-200 \mathrm{kHz}$. A comparative pulse burst of 3 times $5 \mathrm{~ns}$ pulses with $5 \mathrm{~ns}$ seperation between each pulse was also tested to analyze nanosecond pulse burst mechanisms where pulse seperation is an order of magnitude higher than the pulse repetition rate. The resulting temporal profiles used in this work are shown in figure 1. Calibration of the pulse energy was done to equal the same total energy regardless of the pulse shape. This choice of calibration, results in different peak intensities in different pulse shape. 

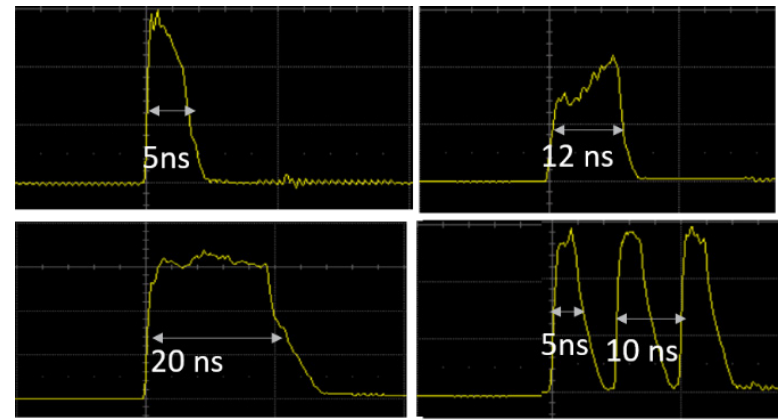

Fig. 1 Temporal pulse shapes used in this study as measured with photodiode on an oscilloscope. Pulse widths are annotated for each pulse, amplitude is arbitrary.

\section{Laser Drilling}

Laser drilling was carried out with an experimental setup for laser micromachining. From the laser source, the beam propagates through a beam expander followed by galvoscanning mirrors and a $100 \mathrm{~mm}$ F-theta scanning lens achieving a spot diameter of $16 \mu \mathrm{m}$ at focus, see figure 2 . Via formation was done using percussion drilling mode only. Sets of vias were drilled while varying the pulse energy, number of pulses, and pulse temporal shape.

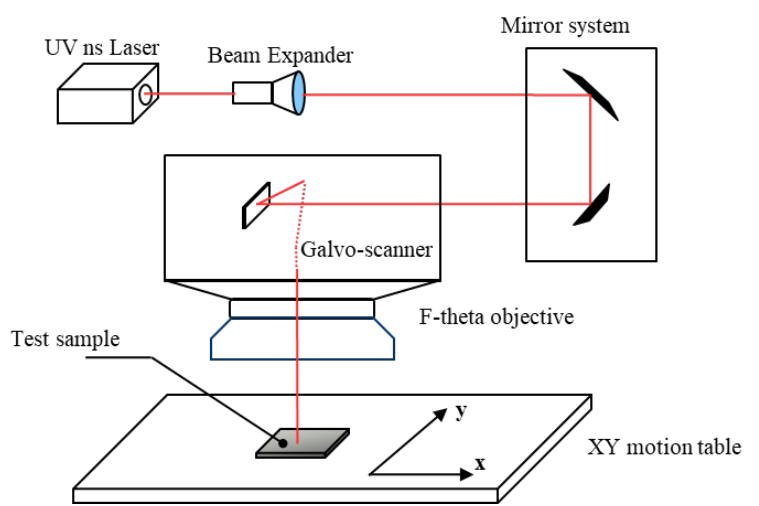

Fig. 2 Laser drilling experimental setup with beam expander, galvo scanning mirrors and f-theta lens for low aberrations with a large field of view.

\section{Resolving of temporal ablation dynamics}

In parallel, a second setup was used for providing highspeed imaging of the ablation process by implementing a pump-probe setup with an $11 \mathrm{~ns}$ pulsewidth laser diode (Osram SPL LL90_3) as the probe. The delay between pump and probe is varied consecutively.

Several modular variations of the setup allow for different observations in a goal to capture the physical process from several aspects. This is illustrated in figure 3 and uses the laser diode for pump-probe microscopy with $905 \mathrm{~nm}$ bandpass filter or with not prove and replacing the bandpass filter with a 450nm LPF (Long Pass Filter) for integration of the plasma while filtering out the laser UV light.

In addition, the placement of photodetectors with filters were used to record the optical intensity signals of the incident, reflected, and scattered light (see figure 4). The copper plasma emission signal was measured by considering the placement of peak intensity in the spectrum typical of ablated copper[17,18], using a $450 \mathrm{~nm}$ LPF to differentiate between the laser $343 \mathrm{~nm}$ UV light and plasma emission lines.
Other tools used for post process analysis included a Bruker 3D interferometer profiler and scanning electron microscope to measure and image the resulting vias from the ablation process.

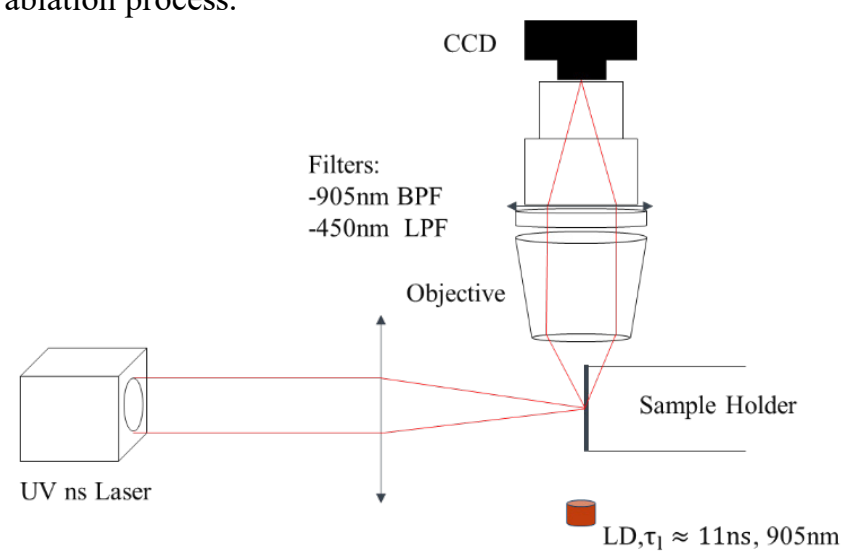

Fig. 3 High-speed imaging setup. Filters were altered for measuring as pump-probe with a laser diode vs. integrated plasma images.

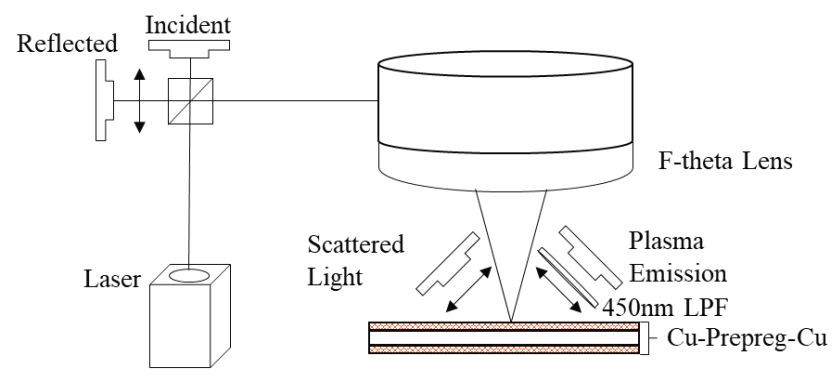

Fig. 4 Plasma Emission sensing by measurement of signals with photodetectors. Each photodetector is shown titled with the intended measured signal.

\section{Material and Calculations}

The materials used in this study consist of three layers, typical for FPCBs, a top layer of $12 \mu \mathrm{m}$ electroplated copper, a middle layer of $25 \mu \mathrm{m}$ of prepreg consisting of epoxy resin with interweaved glass fibers of $5 \mu \mathrm{m}$ diameters, and a bottom $12 \mu \mathrm{m}$ electroplated copper sheet. In addition, a single electroplated copper sheet of $70 \mu \mathrm{m}$ thickness was used to measure ablation thresholds by fitting the diameter squared model[19].

$$
D^{2}=2 \omega_{0} \cdot \ln \left(\frac{\phi}{\phi_{0}}\right)
$$

To determine specific ablation rates, the total crater depth was divided by the number of pulses for each instance.

\section{Results and Discussion}

The most basic measurement comparison for laser ablation is to determine the fluence threshold for substantial material ablation. The fluence values are defined as the averaged value over the beam diameter for a single shot. This measurement was done by analyzing the crater diameters of 100 consecutive pulses at various pulse energies for each pulse shape while the beam diameter is constant. Results of this measurement shown in figure 5 present the determined fluence thresholds and effective waist values evaluated with eq. (1). The thresholds follow the behavior of increasing with pulsewidth, roughly $\phi_{0} \propto$ $\sqrt{\tau}$ [20], where the pulse burst have values ordering it 
between 15 and 20 nanosecond pulsewidths. Apparent is the variation with PRR, showing that pulse to pulse interactions effect the fluence thresholds and effective waist. For the fitting of eq. (1) to be within standard errors, the waist values required being set as a free parameter. The result of this shown in figure 5 is that this value gives a more accurate parameter for the heat affected zone in each case. As the study [19] was done with picosecond pulses where the heat affected zones as minimal, this seems an accurate assumption to make. Interestingly, the shorter pulses show much stronger pulse to pulse interactions at higher PRRs (see following explanations of plasma shielding).

A visual analysis of the via formations done with a scanning electron microscope (SEM) is show in figure 6 . The results show the penetration of top copper layer in FPCB. The lower fluence of $10 \mathrm{~J} / \mathrm{cm}^{2}$ in the top row appear to be above the ablation threshold for all pulsewidths, however the ablation rate is visually lower for the shorter 5ns pulsewidth. This is seen by after 10 pulses; full penetration of the top copper layer was not achieved. In contrast, the pulse burst of $3 \times 5 n$ after 20 pulses reveals the underlying glass fibers within the prepreg layer.

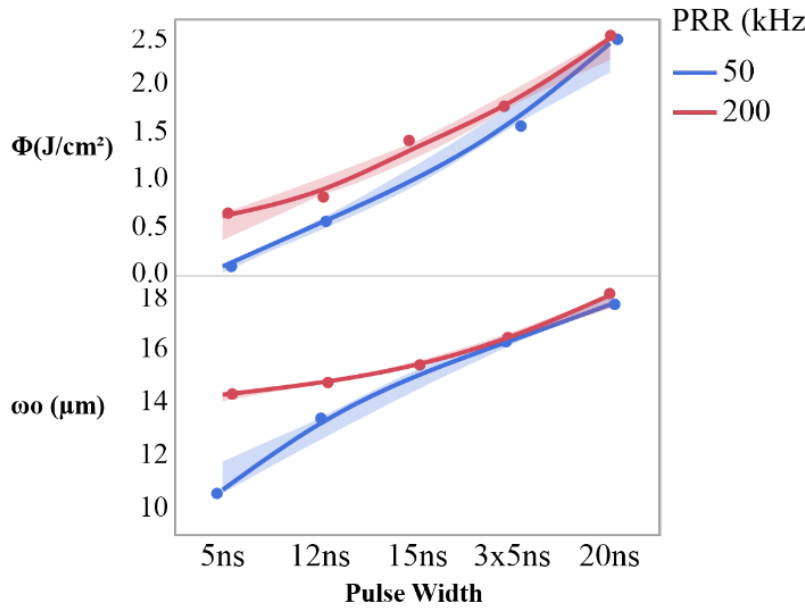

Fig. 5 Ablation fluence thresholds on electroplated copper for different pulse shapes and PRRs. Fitted results produce the fluence threshold value (above) and the effective beam waist (below) for each case.

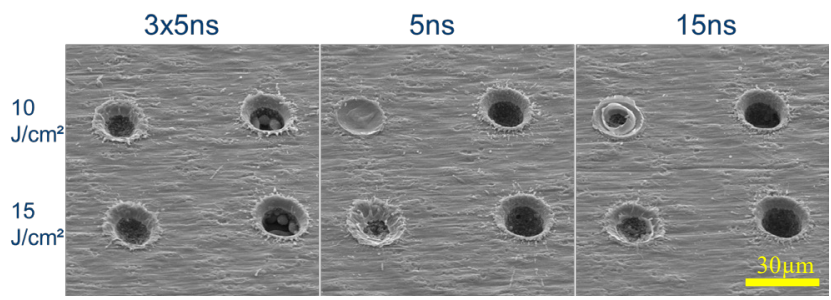

$\mathrm{N}: \quad 10$

20

Fig. 6 SEM image comparison of three different pulsewidths at 200 $\mathrm{kHz}$ PRR, left to right: Pulse burst of $3 \times 5 \mathrm{~ns}$ pulses, and single pulses of $5 \mathrm{~ns}$ and $15 \mathrm{~ns}$. SEM oblique angle was $30^{\circ}$. As noted rows are different fluence values. Each pulse shape columns are different number of total pulses.

Plotting the average ablation rates (of copper top layer) from each set of measurements as described in section 2 are presented in figure 7 . The longer pulses dominate with greater ablation rates only after passing the substantial ablation threshold (as seen lower for shorter pulsewidths). If comparing the $3 \times 5 \mathrm{~ns}$ pulse burst to $5 \mathrm{~ns}$ single pulse, the overall average increase in ablation rate exceeds $40 \%$. Interesting is effect of increasing PRR which reduces the penetration depth for all cases other than for the pulse burst. This decrease for single pulses at high PRRs is shown to be due to the accumulated plasma shielding.

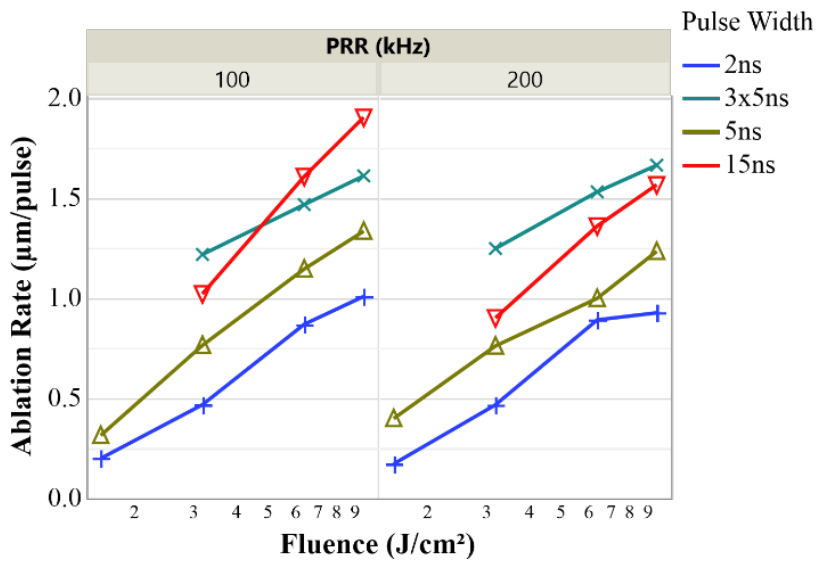

Fig. 7 Ablation rates for different pulse shapes and PRRs.

Imaging of the integrated plasma as show in figures 8 and 9 show the pulse to pulse interactions increasing for higher PRRs, while the pulse burst maintains a much more confined plasma. When considering the intensity for significant plasma shielding[12], the shorter pulses of 5ns produce a much higher peak power of $5 \cdot 10^{9}\left(\mathrm{~W} / \mathrm{cm}^{2}\right)$ as compared to $\sim 1.5 \cdot 10^{9}\left(\mathrm{~W} / \mathrm{cm}^{2}\right)$. However, as seen in the images below, the plasma signal continues to increase for consecutive pulses even with spacings of several microsecond between the pulses for both short and long nanosecond pulses. The significance of this is that for high PRRs one must consider the plasma accumulation between pulses even if the peak power is well below the plasma shielding threshold.

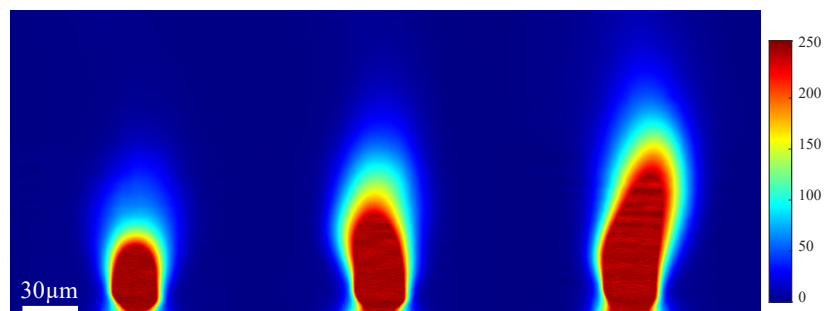

Fig. 8 Images of plasma integration over 20 consecutive pulses of $20 \mathrm{~ns}$ pulsewidths with a fluence of $15 \mathrm{~J} / \mathrm{cm}^{2}$ at PRRs of (left to right): 50, 100, $200 \mathrm{kHz}$. Color map is representative of light intensities of the plasma scaled by intensity as measured on camera sensor.

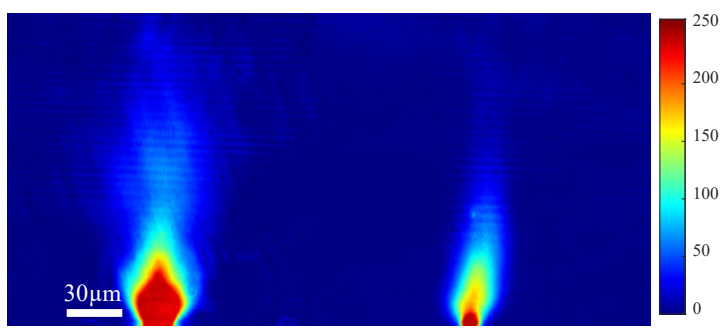

Fig. 9 Images of plasma integration over 10 consecutive pulses with fluence of $15 \mathrm{~J} / \mathrm{cm}^{2}$ at a PRR of $200 \mathrm{kHz}$ for pulsewidths $5 \mathrm{~ns}$ (left) and pulse burst of $3 \times 5 n$ s (right). 
Figure 9 reveals the significance of the pulse burst, here the total plasma emission signal for a single pulse greatly exceeds that of the pulse bursts. To resolve the plasma emission with a higher temporal resolution for determining the lifetime and pulse to pulse interactions, the intensity of the signal was recorded with optical detectors for three different pulsewidths, see figure 10 .

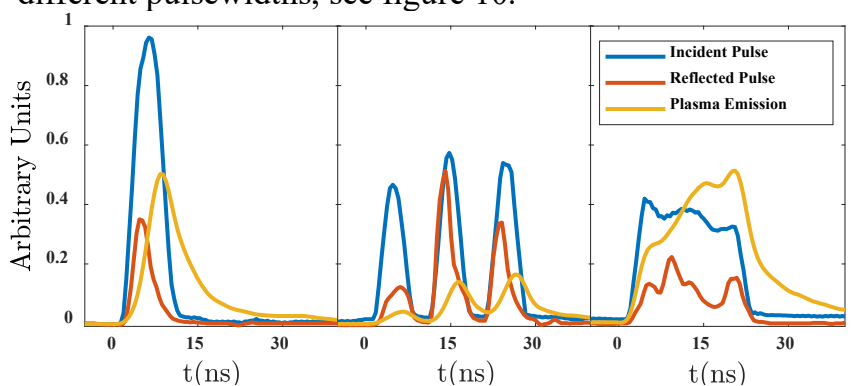

Fig. 10 Plasma emission signals acquired with the setup in figure 4 from left to right of $5 \mathrm{~ns}, 3 \times 5 \mathrm{~ns}$, and $20 \mathrm{~ns}$ pulse shapes. Total laser pulse fluence was $8 \mathrm{~J} / \mathrm{cm}^{2}$. Scaling is arbitrary but consistent for each measured parameter. Signal was measured for single pulse.

The 5ns pulse shows the reflected light slightly trailing the incident pulse, where the reflectance drastically decreases when the plasma emission signal intensifies. This is a common result of plasma generation as the incident and scattered light is partially being absorbed by the plasma. The plasma signal also is extended 10-20 ns after the initial pulse ceases. The pulse burst figure 10 (middle) reveals interesting dynamics. The initial pulse, with a much lower peak intensity relative to the $5 \mathrm{~ns}$ pulse, does not generate a substantial plasma signal, therefore no decrease in the reflected intensity is noted. However, there is enough time between pulses for excitation to occur with a modified impact area forming a slight melt pool which results in a much stronger reflectance of the second pulse. Following this the plasma signal slightly intensifies, causing a decrease in reflectance, by building on the previous pulse's signal which has not extinguished. The third pulse already has more significant plasma from previous pulses to cause a shielding effect slightly after impact of the incident pulse. The 20ns pulse, figure 10 (right), has several discontinuities in the reflected signal, several due to the incident pulse profile as seen, and due to a more significant plasma density that also shows a non-steady state. It is important to note for this pulse that ablation rate is highest for the pulse burst, and decreases slightly for the $20 \mathrm{~ns}$, while the $5 \mathrm{~ns}$ pulse achieves the least ablation. We learn from this measurement that although the plasma imaged accumulation is noticeable for the high PRRs (as seen figures 8 and 9) the strong plasma emission decays after several 10 s of nanoseconds. Further measurements such as the plume (emission mixture of debris, particles along with the plasma vapor) is required to accurately resolve the pulse to pulse interactions.

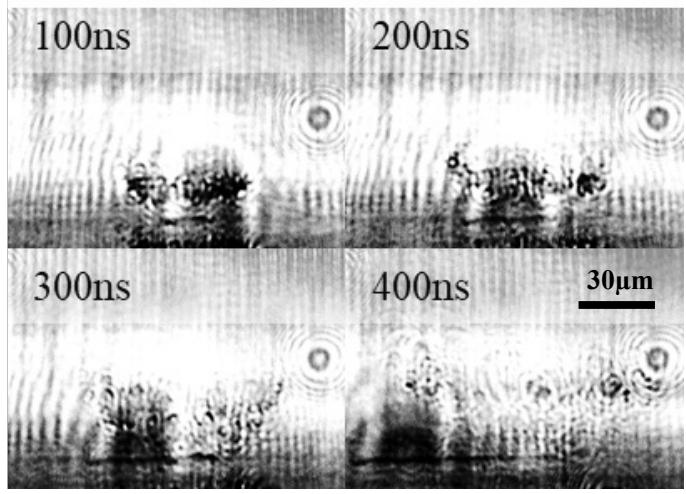

Fig. 11 Imaged ablation plumes from pump-probe setup at intervals following a single incident pulse.

The pump-probe laser setup described in figure 3 was used to acquire the images in figure 11. Analysis of ablation process times showed substantial decrease in plume density after $600 \mathrm{~ns}$ from incident pulse, where some visual debris may stay in the proximity for up to $\sim 1 \mu$ second. This supports the hypothesis that pulse to pulse interactions at 10-100's of $\mathrm{kHz}$ repetition rates are not an exclusive result of initial plasma lifetimes, rather a compound effect resulting from vaporization by sequential pulses of the remaining debris originating from the previous plume in the space above the formed crater.

\section{Conclusions}

Ablation dynamics were analyzed in depth for several nanosecond pulsewidths and pulse bursts, providing further insight on the roles of plasma and plume dynamics as part of the ablation process in microelectronic laser drilling. The confined plasma signal coupled with $\sim 600 \mathrm{~ns}$ for plume dispersion, specifically for the triple pulse burst, proves the possibility of limiting the plasma shielding effects while increasing intensity and pulse repetition rates which may further increase the achievable throughput for micromachining applications.

\section{Acknowledgments}

(1) The authors would like to acknowledge Dr. Roman Vander and Mr. Boris Kling for their professional discussions and advice.

(2) This work received funding from IIA (Israel Innovation Authority), Project No. 65500, "Laser-Matter-Interactions" (LMI).

\section{References}

[1] T. M. Lo and J. S. Young: Int. J. Precision Engin. Manuf., 15, (2014) 1575.

[2] H. Zheng, E. Gan, and G. C. Lim: Opt. Lasers Engin., 36, (2001) 355 .

[3] N. Gorodesky, N. Ozana, Y. Berg, O. Dolev, Y. Danan, Z. Kotler, and Z. Zalevsky: J. Opt., 18, (2016) 095402.

[4] B. Neuenschwander, B. Jaeggi, M. Schmid, and G. Hennig: Phys. Procedia, 56, (2014) 1047.

[5] T. Kramer, S. Remund, M. Chaja, M. Gafner, T. Maehne, and B. Neuenschwander. OSA Laser Appl. Conf., (2019) CW1C-5.

[6] B. Tan: J Micromech. Microeng., 16, (2005) 109. 
[7] S.T. Hendow, R. Romero, S.A. Shakir, and P.T. Guerreiro: Opt. Express, 19, (2011) 10221.

[8] T. Kramer, B. Neuenschwander, B. Jäggi, S. Remund, U. Hunziker, and J. Zürcher: Phy. Procedia, 83, (2016) 123.

[9] T. Hendow, Sami, Shakir, and J. M. Sousa: Proc. SPIE, 7584, (2010) 758417.

[10] K.T. Vu, A. Malinowski, D.J. Richardson, F. Ghiringhelli, L.M.B. Hickey, and M.N. Zervas: Opt. Express, 14, (2006) 10996.

[11] W. T. Hill, and C. H. Lee. "Light-Matter interaction, Atoms and molecules in external fields and nonlinear optics." (Wiley, 2008) 72.

[12] D. Bäuerle, "Laser Processing and Chemistry". (Springer, Berlin, 2011) 237.

[13] G. Raciukaitis, M. Brikas, P. Gecys, and M. Gedvilas: Proc. SPIE, 7005, (2008) 70052L.

[14] F. C. Burns and S. R. Cain. J. Phy. D: Appl. Phys., 29, (1996) 1349.

[15] R. Weber, T. Graf, C. Freitag, A. Feuer, T. Kononenko, and Konov: Opt. Express, 25, (2017) 3966.

[16] F. Di Niso, C. Gaudiuso, T. Sibillano, F.P. Mezzapesa, A. Ancona, and P.M. Lugarà: Opt. Express, 22, (2014) 12200.

[17] K.K. Anoop, S.S. Harilal, R. Philip, R. Bruzzese, and S. Amoruso: J. Appl. Phys., 120, (2016) 185901.

[18] O.A. Ranjbar, A. Omid, L. Zhibin, and A.N. Volkov: Appl. Phys. A, 126, (2020) 1.

[19] J. M. Liu: Opt. Lett., 7, (1982) 196.

[20] B.C. Stuart, M.D. Feit, S. Herman, A.M. Rubenchik, B.W. Shore, and M.D. Perry: J. Opt. Soc. Am., 13, (1996) 459.

(Received: July 6, 2020, Accepted: January 3, 2021) 